\section{Masked Depression}

Robert G. Frank

University of New Mexico, Albuquerque, NM, USA

\section{Synonyms}

Depression equivalent; Depression without a depression; Hidden depression; Vegetative equivalent

\section{Definition}

Masked depression is a term that was largely used in the 1970s and 1980s to refer to patients who complained of somatic symptoms, without any identifiable organic disorder. Depressive symptoms were considered secondary or did not even need to be present. Today this diagnosis does not play a significant clinical or scientific role. Patients today are more likely to be diagnosed with somatization disorder, somatoform disorder, psychosomatic disorder, conversion disorder, neurasthenia, or hypochondriasis.

\section{Cross-References}

Somatization

\section{References and Readings}

Bschor, T. (2002). Masked depression: The rise and fall of a diagnosis. Psychiatrische Praxis, 29, 207-210.

Miodek, A., Szemraj, P., Kocur, J., \& Ryś, A. (2007). Masked depression. Polski Merkuriusz Lekarski, 23, 78-80. 